\title{
From single- to double-first-order magnetic phase transition in magnetocaloric $\mathrm{Mn}_{1-\mathrm{x}} \mathrm{Cr}_{\mathrm{x}} \mathrm{CoGe}$ compounds
}

\author{
N. T. Trung, V. Biharie, L. Zhang, L. Caron, K. H. J. Buschow, and E. Brück ${ }^{a)}$ \\ Fundamental Aspects of Materials and Energy, Faculty of Applied Sciences, TU Delft, Mekelweg 15, \\ 2629 JB Delft, The Netherlands
}

(Received 18 January 2010; accepted 28 March 2010; published online 22 April 2010)

\begin{abstract}
Substitution of some $\mathrm{Cr}$ for $\mathrm{Mn}$ atoms in $\mathrm{MnCoGe}$ was employed to control the magnetic and structural transitions in this alloy to coincide, leading to a single first-order magnetostructural transition from the ferromagnetic to the paramagnetic state with a giant magnetocaloric effect observed near room temperature. Further increase in the $\mathrm{Cr}$ content in the $\mathrm{Mn}_{1-\mathrm{x}} \mathrm{Cr}_{\mathrm{x}} \mathrm{CoGe}$ alloys can induce another first-order magnetoelastic transition from the antiferromagnetic to the ferromagnetic state occurring at lower temperature. The giant magnetocaloric effect as well as the simultaneous tunability of the two magnetic transitions make these materials promising for future cooling applications. (C) 2010 American Institute of Physics. [doi:10.1063/1.3399774]
\end{abstract}

Nowadays, magnetocaloric materials undergoing firstorder magnetic transitions (FOMTs) are intensively investigated because of their potential applications at room temperature. ${ }^{1}$ In contrast to second-order magnetic transitions, FOMTs fall into two categories: magnetostructural transitions (MSTs) and magnetoelastic transitions (METs). In the former case such as $\mathrm{Gd}_{5}\left(\mathrm{Si}_{\mathrm{x}} \mathrm{Ge}_{1-\mathrm{x}}\right)_{4},{ }^{2} \mathrm{MnAs}_{1-\mathrm{x}} \mathrm{Sb}_{\mathrm{x}},{ }^{3}$ and $\mathrm{Ni}_{0.50} \mathrm{Mn}_{0.50-\mathrm{x}} \mathrm{Sn}_{\mathrm{x}},{ }^{4}$ a different crystal structure is found on either side of the magnetic phase transition. As a common feature, the compounds with a MST often show large thermal and field hysteresis. In the case of METs such as $\mathrm{MnFeP}_{1-\mathrm{x}} \mathrm{As}_{\mathrm{x}}$ (Ref. 5) and $\mathrm{La}\left(\mathrm{Fe}_{1-\mathrm{x}} \mathrm{Si}_{\mathrm{x}}\right)_{13},{ }^{6}$ the same crystal structure is found on both sides of the phase transition. However, a distinct lattice distortion accompanied by a possible change in unit-cell volume is observed near the critical transition point. Usually, the compounds exhibiting a MET reveal small or tunable hysteresis. ${ }^{7}$

Earlier studies on MnCoGe confirmed a martensitic structural transformation from the low-temperature orthorhombic (orth.) TiNiSi-type structure (space group Pnma) to the high-temperature hexagonal (hex.) $\mathrm{Ni}_{2} \mathrm{In}$-type structure (space group $\left.\mathrm{P}_{3} / m m c\right)^{8,9}$ On heating, this transformation occurs at the structural transition temperature $\left(T_{\text {str }}\right)$ $\sim 650 \mathrm{~K} .{ }^{9}$ In the stable orth. ground state, $\mathrm{MnCoGe}$ behaves like a typical ferromagnet with a second-order PM-FM transition occurring at the ordering temperature $\left(T_{\mathrm{c}}\right) \sim 345 \mathrm{~K}$, which is far below $T_{\text {str. }}{ }^{10}$ Recently, we have discovered a giant MCE in the magnetic refrigerants based on MnCoGe. ${ }^{11}$ By interstitially adding some boron atoms to $\mathrm{MnCoGe}$, the structural transition at $T_{\mathrm{str}}$ and the magnetic transition at $T_{\mathrm{c}}$ can be controlled to coincide, leading to a single first-order magnetostructural transition (FOMST) from the combined two-phase FM region, in which the orth. TiNiSi- and hex. $\mathrm{Ni}_{2}$ In-type structures coexist, to an almost single-phase PM $\mathrm{Ni}_{2}$ In-type structure. In this paper we shall show that, a single FOMST at $T_{\mathrm{c}}$ and a giant MCE can also be attained by substituting some $\mathrm{Cr}$ for $\mathrm{Mn}$ atoms in $\mathrm{MnCoGe}$. Strikingly, further increase in the $\mathrm{Cr}$ content in $\mathrm{Mn}_{1-\mathrm{x}} \mathrm{Cr}_{\mathrm{x}} \mathrm{CoGe}$ gives rise to the appearance of a second first-order magnetoelastic

${ }^{a)}$ Electronic mail: e.h.brueck@tudelft.nl.
FM-AFM transition at a lower transition temperature $\left(T_{\mathrm{t}}\right)$.

Polycrystalline $\mathrm{Mn}_{1-\mathrm{x}} \mathrm{Cr}_{\mathrm{x}} \mathrm{CoGe} \quad(\mathrm{x}=0.00,0.04,0.11$, $0.18,0.25,0.27$ ) alloys were prepared by arc-melting (see Ref. 11). Shown in Fig. 1(a) are the temperature dependences of the magnetization ( $M-T$ curves) for $\mathrm{x}=0.04,0.11,0.18$, and 0.25 . For $\mathrm{x}=0.04$, the $M-T$ curve shows a single, sharp PM-FM transition at $T_{\mathrm{c}} \sim 322 \mathrm{~K}$. The considerable thermal hysteresis $\left(\Delta T_{\text {hys }}\right)$ clearly evidences the first-order nature of this transition, which is attributed to the intimate coupling between the PM-FM transition and the hex.-orth. structural transition. ${ }^{11}$ For all temperatures below $T_{\mathrm{c}}$, the magnetic moments of this sample are well ordered in the FM state. However, the $M-T$ curves of $\mathrm{x}=0.11,0.18$ and 0.25 indicate that the moments of these materials are more likely ordered in the AFM state at low temperatures. With maintaining the firstorder PM-FM transition at $T_{\mathrm{c}}$, another first-order FM-AFM
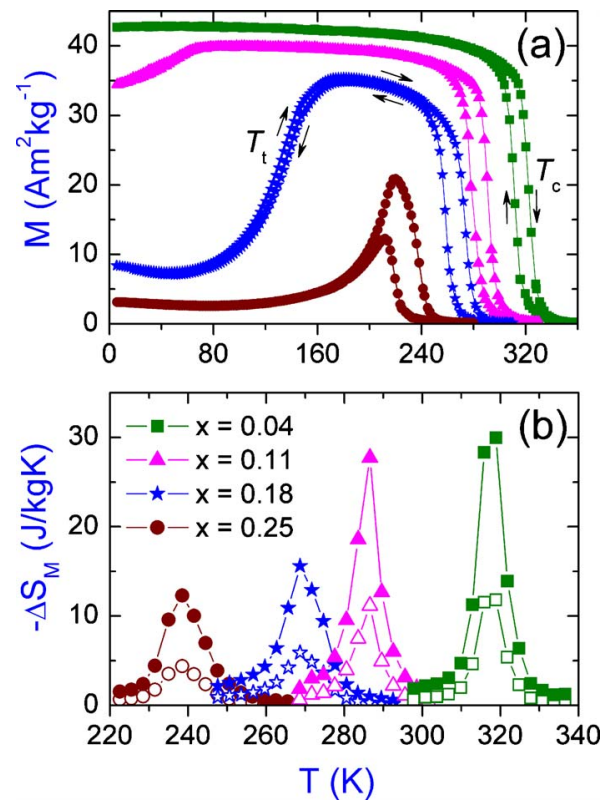

FIG. 1. (Color online) $M-T$ curves measured in a magnetic field $\mathrm{B}=0.1 \mathrm{~T}$ of the $\mathrm{Mn}_{1-\mathrm{x}} \mathrm{Cr}_{\mathrm{x}} \mathrm{CoGe}(\mathrm{x}=0.04,0.11,0.18,0.25)$ alloys (a) and their corresponding $\Delta S_{M}$ at the PM-FM transition under field changes of $\Delta B$ $=0-2 \mathrm{~T}$ (open symbols) and $\Delta B=0-5 \mathrm{~T}$ (closed symbols) (b). 

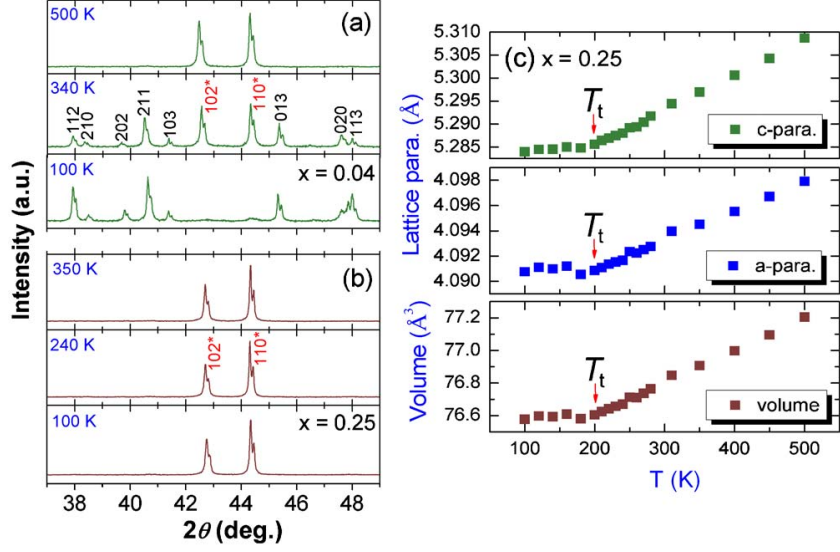

FIG. 2. (Color online) XRD patterns measured in zero-field upon heating at 100,340 , and $500 \mathrm{~K}$ for $\mathrm{x}=0.04$ (a) and at 100,240 , and $350 \mathrm{~K}$ for $\mathrm{x}=0.25$ (b).In Fig. 2(a), the pattern measured at $340 \mathrm{~K}$ confirms the coexistence of the orth. phase ( $h k l$ Miller indices without $*$ ) and the hex. phase ( $h k l$ Miller indices with *) (c) Variation in lattice parameters and volume of the hex. $\mathrm{Ni}_{2}$ In-type unit-cell as a function of temperature for the $\mathrm{x}=0.25$ sample.

transition can thus take place at $T_{\mathrm{t}}$. Note that the double FOMTs at $T_{\mathrm{t}}$ and $T_{\mathrm{c}}$ as in $\mathrm{Mn}_{1-\mathrm{x}} \mathrm{Cr}_{\mathrm{x}} \mathrm{CoGe}$ have not been observed in any other type of martensitic transition materials yet. Strikingly, these two FOMTs are simultaneously tunable. With increasing $\mathrm{Cr}$ doping, $T_{\mathrm{c}}$ is significantly lowered, however, $T_{\mathrm{t}}$ is strongly shifted to higher temperatures. Here, the occurrence of small hysteresis at $T_{\mathrm{t}}$ indicates that the FMAFM transition may have a magnetoelastic character. As can be seen from Fig. 1(a), the substitution of some $\mathrm{Cr}$ atoms has a strong influence on the magnetization in both the FM and AFM phases. The total entropy change $\left(\Delta S_{M}\right)$ of materials with hysteretic first-order transitions can reliably be calculated from the Maxwell relation. ${ }^{12,13}$ Displayed in Fig. 1(b) are the $\Delta S_{M}$ calculated for the PM-FM transition of $\mathrm{Mn}_{1-\mathrm{x}} \mathrm{Cr}_{\mathrm{x}} \mathrm{CoGe}$ (with $\mathrm{x}=0.04,0.11,0.18,0.25$ ). Here all samples exhibit a giant MCE around $T_{\mathrm{c}}$. For $\Delta B=0-5 \mathrm{~T}$, large $\Delta S_{M}$ values of $-28.5 \mathrm{~J} \mathrm{~kg}^{-1} \mathrm{~K}^{-1}$ and $-27.7 \mathrm{~J} \mathrm{~kg}^{-1} \mathrm{~K}^{-1}$ are obtained for the sample with $\mathrm{x}=0.04$ and 0.11 , respectively. When further increasing the $\mathrm{Cr}$ content, the MCE becomes much lower, with $\Delta S_{M}$ values of $-15.6 \mathrm{~J} \mathrm{~kg}^{-1} \mathrm{~K}^{-1}$ and $-12.3 \mathrm{~J} \mathrm{~kg}^{-1} \mathrm{~K}^{-1}$ for $\mathrm{x}=0.18$ and 0.25 , respectively.

The structural properties of $\mathrm{Mn}_{1-\mathrm{x}} \mathrm{Cr}_{\mathrm{x}} \mathrm{CoGe}$ were investigated by $\mathrm{x}$-ray diffraction (XRD). The volume fraction (vol \%) of the orth. and the hex. phase was calculated by Rietveld refinement using the FULLPROF package. ${ }^{11,14}$ Figure 2(a) presents XRD patterns for $\mathrm{x}=0.04$ taken below, near and above $T_{\mathrm{c}}$. At $100 \mathrm{~K}$ we observe predominantly the orth. TiNiSi-type structure with a small fraction of the hex. $\mathrm{Ni}_{2}$ In-type. Obviously, the single hex. $\mathrm{Ni}_{2}$ In-type phase above $T_{\mathrm{c}}$ can largely transform to the orth. TiNiSi-type phase below $T_{\mathrm{c}}$. Indeed, this again verifies the occurrence of a FOMST at $T_{\mathrm{c}}$. In contrast, the XRD patterns presented in Fig. 2(b) for $x=0.25$ show almost single phase of the hex. structure from below $T_{\mathrm{t}}$ to above $T_{\mathrm{c}}$. Only by carefully analyzing the XRD pattern measured below $T_{\mathrm{c}}$, a small amount of orth. phase $(\sim 2.4$ vol \%) is detected. Although the volume change due to the structural transformation is negligibly small, the FOMST at $T_{\mathrm{c}}$ is still evident for this sample. Apparently, the substitution of $\mathrm{Cr}$ for Mn can stabilize the hex. phase relatively to the orth. phase. As shown in Table I, the variation in phase fractions is strongly dependent on the $\mathrm{Cr}$ content. Note that when the $\mathrm{Cr}$ content is slightly higher than 0.25 at. $\%$, as for $\mathrm{x}=0.27$, refinement of the XRD data shows that all reflections can only be indexed on the basis of a single hex. $\mathrm{Ni}_{2}$ In-type phase. Since the magnetostructural coupling can no longer take place and the AFM arrangement is no longer stable, we only observe the second-order PM-FM transition in this sample. ${ }^{15}$ In comparison with $\mathrm{MnCoGeB}_{\mathrm{x}}$, ${ }^{11}$ the properties of $\mathrm{Mn}_{1-\mathrm{x}} \mathrm{Cr}_{\mathrm{x}} \mathrm{CoGe}$ are even more complicated due to the appearance of the FM-AFM transition at $T_{\mathrm{t}}$. In these materials, the lower limit of $T_{\mathrm{c}}$ is always given by $T_{\mathrm{t}}$. As the hex. phase fraction below $T_{\mathrm{c}}$ strongly increases with $\mathrm{Cr}$ doping, the magnetostructural decoupling is only observed for the $\mathrm{x}=0.27$ sample with no structural change.

Another result from the XRD data obtained on $\mathrm{x}=0.18$ and 0.25 confirms that the relative phase fraction ratio between the orth. and hex. structures does not change at $T_{\mathrm{t}}$. Hence, a magnetostructural coupling between the FM-AFM transition and the hex.-orth. transition apparently does not occur. A detailed study of the origin of the first-order magnetoelastic FM-AFM transition was carried out on $\mathrm{x}=0.25$. This sample was chosen because it mostly contains the hex. phase. Thus, the errors that might arise from the coexistence of two structures at $T_{\mathrm{t}}$ can be neglected in our analysis. In Fig. 2(c), the variation in lattice parameters and volume of the hex. $\mathrm{Ni}_{2}$ In-type unit-cell for $\mathrm{x}=0.25$ is displayed. Obviously, at $\sim 200 \mathrm{~K}$ one can observe a kink, which is very close to $T_{\mathrm{t}}$ of the FM-AFM transition. Like in invar alloys, the lattice parameters and the unit-cell volume of $x=0.25$ are almost constant below $T_{\mathrm{t}}$. The increase in these above $T_{\mathrm{t}}$ is consistent with a linear thermal-expansion. Similar to

TABLE I. Variation in magnetic phase transition type, $T_{\mathrm{t}}$ and $T_{\mathrm{c}}$ obtained on heating, $\Delta T_{\mathrm{hys}}$ at $T_{\mathrm{c}}, \Delta S_{M \text {, max }}$ (for $\Delta B=0-5 \mathrm{~T}$ ) of the PM-FM transition, and the phase fraction (vol \%) of the orth. and hex. structures at $100 \mathrm{~K}$ for the $\mathrm{Mn}_{1-\mathrm{x}} \mathrm{Cr}_{\mathrm{x}} \mathrm{CoGe}$ alloys.

\begin{tabular}{|c|c|c|c|c|c|c|c|}
\hline \multirow[b]{2}{*}{ Samples } & \multirow[b]{2}{*}{ Phase transitions } & \multirow{2}{*}{$\begin{array}{c}T_{\mathrm{t}} \\
(\mathrm{K})\end{array}$} & \multirow{2}{*}{$\begin{array}{c}T_{\mathrm{c}} \\
(\mathrm{K})\end{array}$} & \multirow{2}{*}{$\begin{array}{l}\Delta T_{\text {hys }} \\
(\mathrm{K})\end{array}$} & \multirow{2}{*}{$\begin{array}{c}-\Delta S_{M, \max } \\
\left(\mathrm{J} \mathrm{kg}^{-1} \mathrm{~K}^{-1}\right)\end{array}$} & \multicolumn{2}{|c|}{$\begin{array}{c}\text { Phase fraction } \\
\text { (vol \%) }\end{array}$} \\
\hline & & & & & & Orth. & Hex. \\
\hline$x=0.00$ & Second-order PM-FM & & 345 & 0 & 4.8 & 100.0 & 0.0 \\
\hline$x=0.04$ & First-order PM-FM & $\sim 0$ & 322 & 10 & 28.5 & 96.9 & 3.1 \\
\hline $\mathrm{x}=0.11$ & First-order PM-FM-AFM & 43 & 292 & 12 & 27.7 & 89.4 & 10.6 \\
\hline $\mathrm{x}=0.18$ & First-order PM-FM-AFM & 133 & 274 & 15 & 15.6 & 29.2 & 70.8 \\
\hline$x=0.25$ & First-order PM-FM-AFM & 212 & 237 & 20 & 12.3 & 2.4 & 97.6 \\
\hline$x=0.27$ & Second-order PM-FM & & 190 & 0 & 1.8 & 0.0 & 100.0 \\
\hline
\end{tabular}


$\mathrm{Ni}-\mathrm{Mn}-\mathrm{In},{ }^{12}$ it is reasonable to assume that, for the sample with $0<\mathrm{x} \leq 0.25$, the smaller distance between the $\mathrm{Mn}$ and Co sublattices below $T_{\mathrm{t}}$ favors the AFM state whereas the relatively larger distance above $T_{\mathrm{t}}$ is suitable for the FM arrangement of the magnetic moments.

Because the contribution of electronic entropy change $\left(\Delta S_{E}\right)$ is very small, the $\Delta S_{M}$ for a FOMST mainly consists of the spin-entropy change $\left(\Delta S_{\text {spin }}\right)$ plus the lattice-entropy change $\left(\Delta S_{L}\right){ }^{16}$ For the martensitic transition in Ni-Mn-In alloys, the contribution of $\Delta S_{L}$ has been estimated at about $50 \%$ of $\Delta S_{M} \cdot{ }^{12}$ For $\mathrm{Gd}_{4} \mathrm{Si}_{\mathrm{x}} \mathrm{Ge}_{4-\mathrm{x}}$, this contribution is found to

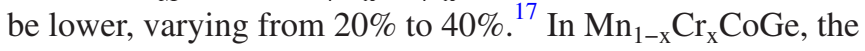
amount of orth. phase below $T_{\mathrm{c}}$ is strongly reduced with increasing the $\mathrm{Cr}$ content (see Table I). For $\mathrm{x}=0.25, \Delta S_{L}$ involving the transformation from the high-symmetry hex. to the low-symmetry orth. structure is negligibly small. The giant MCE found in this compound is attributed to a dominant contribution of the $\Delta S_{\text {spin }}$ [see Fig. 1(b)]. Actually, the two FOMTs at $T_{\mathrm{t}}$ and $T_{\mathrm{c}}$ imply both a negative and a positive $\Delta S_{M}$ in $\mathrm{Mn}_{1-\mathrm{x}} \mathrm{Cr}_{\mathrm{x}} \mathrm{CoGe}$. Although the MCE from the FMAFM transition is small (for example for $\mathrm{x}=0.18, \Delta S_{M}$ $\sim 1.2 \mathrm{~J} \mathrm{~kg}^{-1} \mathrm{~K}^{-1}$ for $\Delta B=0-5 \mathrm{~T}$ ) in comparison with that from the PM-FM transition, improvement of the MCE magnitude of both these FOMPTs can be utilized to enhance the efficiency of a special type of magnetic refrigerators. ${ }^{18}$

In summary, the substitution of $\mathrm{Cr}$ for $\mathrm{Mn}$ atoms has been employed to tailor the magnetic and structural transitions in the magnetocaloric $\mathrm{Mn}_{1-\mathrm{x}} \mathrm{Cr}_{\mathrm{x}} \mathrm{CoGe}$ compounds. $\mathrm{By}$ increasing the $\mathrm{Cr}$ content, we can introduce a first-order magnetostructural PM-FM transition at $T_{\mathrm{c}}$ and another first-order magnetoelastic FM-AFM transition at $T_{\mathrm{t}}$. The occurrence of these double FOMTs in a single material and the possible extraction of the concomitant $\Delta S_{L}$ from the $\Delta S_{M}$ are extremely interesting for further studies on critical phase transitions. The giant MCEs as well as the simultaneous tunabil- ity of the two magnetic transitions can make these materials promising for future cooling applications.

Special thanks are due to M. P. Steenvoorden for his assistance on XRD experiments. This work was financially supported by the Dutch Technology Foundation (STW) and the Foundation for Fundamental Research on Matter (FOM), which is financially supported by the Netherlands Organisation for Scientific Research (NWO).

${ }^{1}$ E. Brück, J. Phys. D 38, R381 (2005).

${ }^{2}$ V. K. Pecharsky and K. A. Gschneidner, Phys. Rev. Lett. 78, 4494 (1997).

${ }^{3}$ H. Wada and Y. Tanabe, Appl. Phys. Lett. 79, 3302 (2001).

${ }^{4}$ T. Krenke, E. Duman, M. Acet, E. F. Wassermann, X. Moya, L. Mañosa, and A. Planes, Nature Mater. 4, 450 (2005).

${ }^{5}$ O. Tegus, E. Brück, K. H. J. Buschow, and F. R. de Boer, Nature (London) 415, 150 (2002).

${ }^{6}$ F. X. Hu, B. G. Shen, J. R. Sun, Z. H. Cheng, G. H. Rao, and X. X. Zhang, Appl. Phys. Lett. 78, 3675 (2001).

${ }^{7}$ N. T. Trung, Z. Q. Ou, T. J. Gortenmulder, O. Tegus, K. H. J. Buschow, and E. Brück, Appl. Phys. Lett. 94, 102513 (2009).

${ }^{8} \mathrm{O}$. Beckman and L. Lundgren, in Handbook of Magnetic Materials, edited by K. H. J. Buschow (Elsevier, New York, 1991), Vol. 6, Chap. 3.

${ }^{9}$ T. Kanomata, H. Ishigaki, T. Suzuki, H. Yoshida, S. Abe, and T. Kaneko, J. Magn. Magn. Mater. 140-144, 131 (1995).

${ }^{10}$ S. Lin, O. Tegus, E. Brück, W. Dagula, T. J. Gortenmulder, and K. H. J. Buschow, IEEE Trans. Magn. 42, 3776 (2006).

${ }^{11}$ N. T. Trung, L. Zhang, L. Caron, K. H. J. Buschow, and E. Brück, Appl. Phys. Lett. (to be published).

${ }^{12}$ B. Li, W. J. Ren, Q. Zhang, X. K. Lv, X. G. Liu, H. Meng, J. Li, D. Li, and Z. D. Zhang, Appl. Phys. Lett. 95, 172506 (2009).

${ }^{13}$ L. Caron, Z. Q. Ou, T. T. Nguyen, D. T. Cam Thanh, O. Tegus, and E. Brück, J. Magn. Magn. Mater. 321, 3559 (2009).

${ }^{14}$ J. Rodríguez-Carvajal, Physica B 192, 55 (1993).

${ }^{15}$ See supplementary material at http://dx.doi.org/10.1063/1.3399774 for Phase diagram of the $\mathrm{Mn}_{1-x} \mathrm{Mn}_{x} \mathrm{CoGe}$ compounds as derived from the magnetic and structural measurements.

${ }^{16}$ V. K. Pecharsky, A. P. Holm, K. A. Gschneidner, Jr., and R. Rink, Phys. Rev. Lett. 91, 197204 (2003).

${ }^{17}$ G. J. Liu, J. R. Sun, J. Lin, Y. W. Xie, T. Y. Zhao, H. W. Zhang, and B. G. Shen, Appl. Phys. Lett. 88, 212505 (2006).

${ }^{18}$ X. Zhang, B. Zhang, S. Y. Yu, Z. H. Liu, W. J. Xu, G. D. Liu, J. L. Chen, Z. X. Cao, and G. H. Wu, Phys. Rev. B 76, 132403 (2007) 